\title{
Determinants of Hospital Inpatient Costs in the Iranian Elderly: A Micro-costing Analysis
}

\author{
Ebrahim Hazrati' ${ }^{1}$ Zahra Meshkani², Saeed Husseini Barghazan², Sanaz Zargar Balaye Jame ${ }^{3}$, \\ Nader Markazi-Moghaddam ${ }^{3}$ \\ ${ }^{1}$ Department of Anesthesiology and Critical Care, AJA University of Medical Sciences, Tehran, Iran; ${ }^{2}$ Health Management and Economics Research \\ Center, Iran University of Medical Sciences, Tehran, Iran; ${ }^{3}$ Department of Health Management and Economics, School of Medicine, AJA University of \\ Medical Sciences, Tehran, Iran
}

Objectives: Aging is assumed to be accompanied by greater health care expenditures. The objective of this retrospective, bottom-up micro-costing study was to identify and analyze the variables related to increased health care costs for the elderly from the provider's perspective.

Methods: The analysis included all elderly inpatients who were admitted in 2017 to a hospital in Tehran, Iran. In total, 1288 patients were included. The Mann-Whitney and Kruskal-Wallis tests were used.

Results: Slightly more than half (51.1\%) of patients were males, and $81.9 \%$ had a partial recovery. The 60-64 age group had the highest costs. Cancer and joint/orthopedic diseases accounted for the highest proportion of costs, while joint/orthopedic diseases had the highest total costs. The surgery ward had the highest overall cost among the hospital departments, while the intensive care unit had the highest mean cost. No statistically significant relationships were found between inpatient costs and sex or age group, while significant associations $(p<0.05)$ were observed between inpatient costs and the type of ward, length of stay, type of disease, and final status. Regarding final status, costs for patients who died were 3.9 times higher than costs for patients who experienced a partial recovery. Conclusions: Sex and age group did not affect hospital costs. Instead, the most important factors associated with costs were type of disease (especially chronic diseases, such as joint and orthopedic conditions), length of stay, final status, and type of ward. Surgical services and medicine were the most important cost items.

Key words: Aging, Hospitals, Hospital costs, Costs and cost analysis, Economics

\section{INTRODUCTION}

Living longer is one of the most remarkable human achievements over the past century. Successes in both preventive and

Received: September 4, 2019 Accepted: April 4, 2020

Corresponding author: Nader Markazi-Moghaddam, MD, PhD Department of Health Management and Economics, School of Medicine, AJA University of Medical Science, West Fatemi Avenue, Tehran 009821141178541, Iran

E-mail: nmmoghaddam@gmail.com

This is an Open Access article distributed under the terms of the Creative Commons Attribution Non-Commercial License (https://creativecommons.org/licenses/bync/4.0/) which permits unrestricted non-commercial use, distribution, and reproduction in any medium, provided the original work is properly cited. curative medicine and widespread technological advances have resulted in a steady increase in life expectancy [1], which in turn has led to worldwide population aging. The proportion of the elderly is expected to rise from $10 \%$ to $22 \%$ globally $[2,3]$. Diseases and disabilities are considered to be significant challenges for the elderly [4]. The medical expenses of seniors in the last 10 years have risen three-fold [5]. The burden of disease in the elderly is associated with cardiovascular disease; malignant neoplasms; chronic respiratory conditions; diabetes; and joint, mental, neurological, infectious/parasitic, and cognitive diseases [6].

Population aging influences the reimbursement system in healthcare. The elderly population drives demand for certain 
needs due to aging-associated diseases, and this issue poses challenges to the health sector [7]. In most countries, the elderly are covered by medical and hospital services and pay lower out-of-pocket expenses for utilizing these services; however, meaningful differences among countries relate to the coverage of medications. The United States, for example, has implemented an insurance policy known as Medicare for the elderly [8]. According to Chan et al. [9], the costs of elderly patients in Singapore were more than expected. The financing schemes in Singapore reduced out-of-pocket payments by the elderly to some extent; however, financial support is particularly needed to compensate for out-of-pocket payments by ill elderly individuals who are not in good financial condition.

There is no standard numerical criterion mandated by the United Nations (UN), but the UN-agreed cut-off to refer to the older population is more than 60 years [10]. According to the census conducted by the Statistical Center of Iran, it has been projected that the total population of Iran will reach 85 million by 2021 , of whom approximately 10 million ( $8.5 \%$ ) will be elderly. Given the increase in the prevalence of chronic diseases and the incidence of many disabilities among the elderly, healthcare for this group can impose heavy costs on these individuals, their families, and the health system (including insurance companies); this issue requires attention and timely planning. One reason for increasing healthcare expenditures is the increasing age of the population. Although policy-makers cannot stop population aging, it is important that they anticipate the health services needs of the elderly and the fiscal impacts of these demographic changes [11].

Several studies, especially in European countries, have investigated the magnitude of the impact of aging on healthcare expenditures. Aging is one of the most significant drivers of health costs in the United States population, as the medical costs of the elderly are 3-5 times higher than those of younger people because of their increasing health needs [12]. Brockmann [13] showed that the costs of caring for people in their last year of life accounted for a higher percentage of health expenditures in Germany in 2002. Based on the understanding that aging leads to increased hospitalization costs, Hazra et al. [14] analyzed healthcare costs in the senior elderly in the United Kingdom with the goal of addressing the empirical gap in the literature concerning the trajectory of healthcare costs in the over80 s. The demographic transition has been considered as a major factor contributing to the costs of healthcare, although little attention has been paid to it. A possible reason for this is that identifying clear relationships among demographic changes, diseases, and costs is challenging. Therefore, we intended to conduct such an analysis for a hospital as a representative of the main type of institutional provider of healthcare.

Micro-costing analyses collect detailed data on resource utilization and the value of those utilized resources. Micro-costing analysis results can be presented as stand-alone studies or can be input directly into health economic evaluation analyses, such as cost-effectiveness analyses [15]. Polder et al. [16] showed that the healthcare costs in the last year of life were 13.5 times higher than the mean lifetime healthcare costs for a person, with costs of $€ 14906$ in the last year of life. Furthermore, hospital care accounted for $54 \%$ of all these costs.

We, therefore, investigated hospital costs as a part of healthcare costs according to diagnosis, age, and sex to determine which illnesses and demographic categories in the elderly were associated with the greatest demands for hospital care. In light of the demographic changes that Iran will face in the future, this research is expected to be helpful for planning, allocating funds, and taking into account the needs of the elderly.

\section{METHODS}

\section{Data Source}

Data were obtained from a teaching hospital using the Iranian hospital information system along with daily hospital admissions data. Hospital records were based on International Classification of Diseases, 10th revision codes. In order to prevent duplicate calculations, the final list of costs was considered. The selected hospital was a general hospital with 190 inpatient beds capable of accepting all types of patient admissions located in the center of Tehran, the capital city of Iran. This hospital was chosen based on availability and accessibility of the data.

\section{Study design}

This was a retrospective, descriptive costing study. A bottom-up micro-costing analysis was performed in order to analyze the data.

\section{Sampling}

The study was conducted among all elderly patients admitted to the hospital. All final bills in elderly patients' medical records from the fiscal year of 2017 were included. Therefore, no sampling was required. The data were registered in a designed form. Sex, age, Para clinical service costs, final status (recovery 
or death), cost of medications and drugs, and the type of inpatient ward were recorded.

\section{Costing analysis}

A bottom-up approach was implemented. First, all cost items were identified. Second, all of the services provided to the elderly, such as surgical services, drugs, and medical tools, and paraclinical services (laboratory tests, magnetic resonance imaging, etc.) were considered. The elderly were divided into 6 age groups, starting at 60 years. The frequency, standard deviations, mean, minimum, and maximum costs were compared among the groups. Finally, we assessed the relationships between hospital inpatient costs and age, sex, type of disease, length of stay, ward, and final status. Furthermore, costs were categorized into 14 sections based on data in patients' medical records. All costs were converted into US dollars (US\$) using the exchange rate from 2017 (one US $\$=42000$ rials) that was reported by the central bank of Iran.

\section{Statistical Analysis}

Data were assessed for normality using the KolmogorovSmirnov test. Since this test showed data a non-normal distribution $(p<0.05)$, we used the Mann-Whitney test to evaluate the relationship between sex and costs and the Kruskal-Wallis test to evaluate the relationship between age group and costs. Stata version 13.0 (StataCorp., College Station, TX, USA) was used for the statistical analysis.

\section{Ethics Statement}

This study was approved by the AJA University in 2016 (registration code: 593191), but at that time studies that did not deal with the patient did not require ethical code.

\section{RESULTS}

Of the 1288 elderly inpatients, $48.8 \%(n=629)$ were females, and their mean hospital stay was 7.7 days. The participants ranged in age from 60 years to 101 years, with a mean age of $72.63 \pm 8.68$ years. The $60-64$ years age group accounted for the highest percentage of patients (22.2\%). Costs were classified into 14 items, as presented in Table 1. Surgical operations, drugs and medical tools, and dialysis services had the highest average costs, of US\$294, US\$207, and US\$195, respectively. The mean cost per elderly patient was US\$637. The minimum and maximum hospitalization costs of the elderly patients in
Table 1. Mean expenditures for cost items for elderly hospitalized patients (US\$)

\begin{tabular}{lc}
\hline Cost items & Mean cost (\%) \\
\hline Visit $^{1}$ & $8(0.8)$ \\
Consultations and nursing & $41(4.2)$ \\
Surgical services $^{2}$ & $294(29.9)$ \\
Drugs and medical tools & $207(21.1)$ \\
Radiology & $8(0.8)$ \\
Sonography & $14(1.4)$ \\
Magnetic resonance imaging & $41(4.2)$ \\
Computed tomography scans & $21(2.1)$ \\
Laboratory and pathology $^{\text {Chemotherapy }}$ & $45(4.6)$ \\
Electromyography & $49(5.0)$ \\
Dialysis services & $24(2.4)$ \\
Rehabilitation & $195(19.9)$ \\
Nonsurgical services & $8(0.8)$ \\
\hline
\end{tabular}

US\$, US dollar.

${ }^{1}$ Calculated per occupied bed-days.

${ }^{2}$ Calculated for patients who underwent surgery.

the hospital were $\$ 21$ and $\$ 13166$, respectively (Table 2 ).

In total, $22.4 \%$ of the total costs were accounted for by patients in the age group of $60-64$ years, $17.5 \%$ by those in the age group of $65-69$ years, $17.3 \%$ by those in the age group of $70-74$ years, $19.7 \%$ by those in the age group of $75-79$ years, $12.3 \%$ by those in the age group of $80-84$ years, and $10.9 \%$ by those in the age group of 85 years and over. The highest and lowest proportions of total costs were accounted for by the age groups of 60-64 years and 85 years and over, respectively.

The Mann-Whitney test showed no statistically significant relationship between inpatient costs and sex $(p=0.38)$, and the Kruskal-Wallis test showed no statistically significant relationship between the costs of hospitalization and age group $(p=0.06)$. Death occurred in 39 cases $(3.0 \%)$. The mean cost of these cases was US\$1768, which was 3.9 times higher than for patients discharged with partial recovery. A statistically significant relationship was observed between individuals' final status and inpatient costs $(p<0.001)$ (Table 2$)$.

The most frequent diseases were cancer and joint/orthopedic diseases, which accounted for $22.9 \%$ and $15.9 \%$ of cases, respectively. The mean cost per cancer patient was US\$409, and that for patients with joint/orthopedic conditions was US\$680. A statistically significant relationship was found between the type of disease and hospitalization costs, as well as a statistically significant association between the type of ward and hospitalization costs $(p<0.001)$. Patients with a length of 
Table 2. Details on the costs of elderly hospitalized patients (US\$)

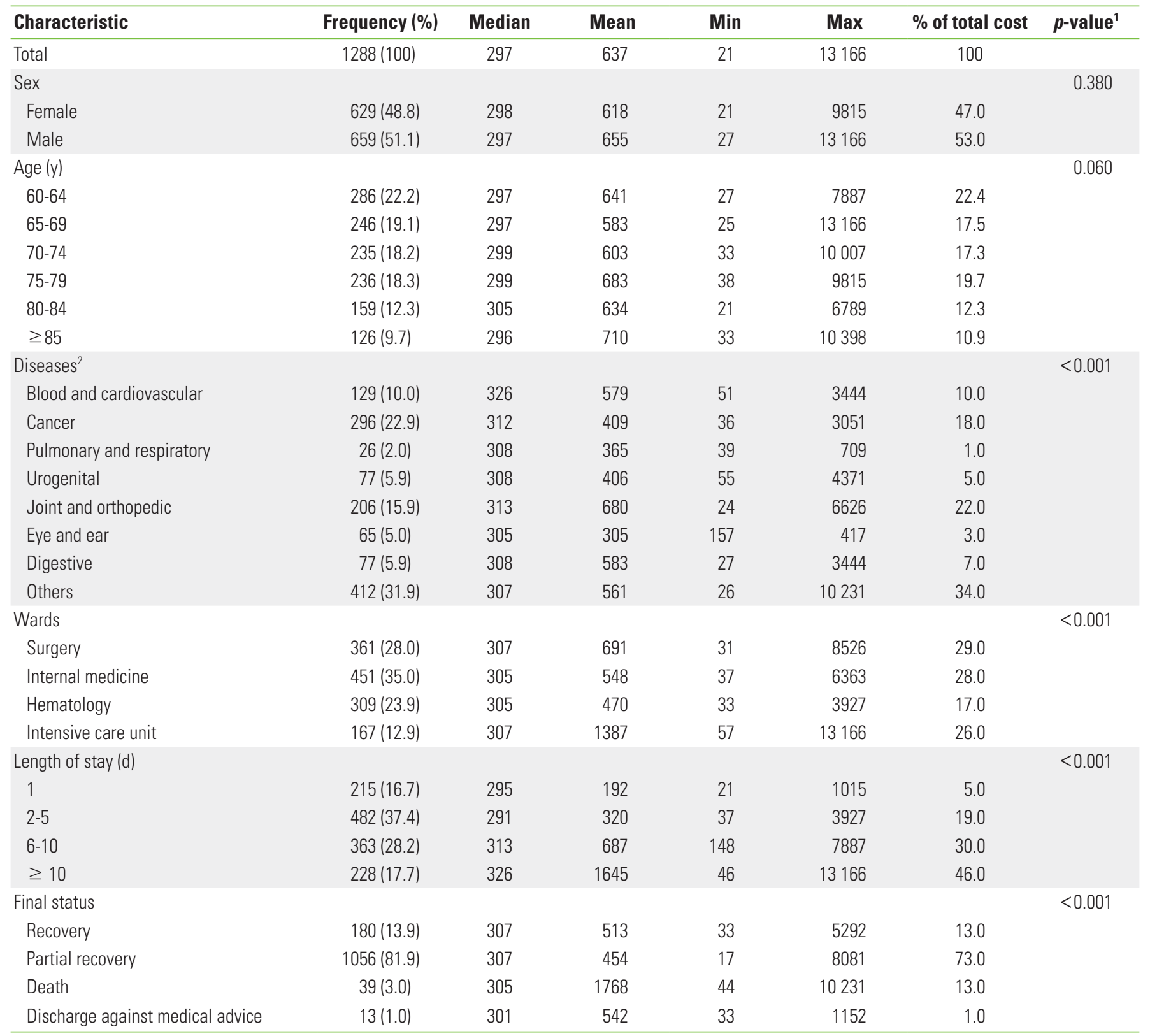

US\$, US dollar; Min, minimum; Max, maximum. ${ }^{1} p$-values are for mean costs.

${ }^{2}$ The disease categories were based on International Classification of Diseases, 10th revision codes.

stay of more than 10 days had higher mean costs (US\$1645) than those with shorter stays. There was a statistically significant relationship between the length of stay and inpatient costs $(p<0.001)$.

The surgical and internal medicine wards had the highest percentages of total costs ( $28.0 \%$ and $35.0 \%$, respectively). However, the intensive care unit had the highest mean costs (US\$1387) (Table 2).

\section{DISCUSSION}

The presented data confirm that the costs of care were higher in patients facing death. The lowest overall proportion of hospitalization costs was found for patients who were 85 years of age and over. Nonetheless, this group had the highest average hospital costs, in line with previous studies such as that of Mcgrail et al. [17], who showed an additional cost of 
dying even accounting for age-related increases in social and nursing costs. Polder et al. [16] obtained a similar result in a study conducted in Denmark. In their study, the costs incurred by persons approaching death were 13.5 times higher than those of other individuals, and $54 \%$ of costs were associated with hospital care, whereas only $19 \%$ of them were related to nursing care at home. They showed that the highest costs were related to cancer, and the lowest costs were related to myocardial infarction [6]. In our study, cancer and joint/orthopedic diseases accounted for the highest proportions of costs ( $22.9 \%$ and $15.9 \%$, respectively), while joint/orthopedic diseases had the highest mean cost. This was mainly because patients with joint diseases spent more days in the hospital. Therefore, the results of our study do not match those reported by Polder et al. [16]

Individuals approaching death utilized an increased amount of medical services, including medications. Kildemoes et al. [18] investigated the effects of age on medication costs in Denmark. They considered current trends in medication use and estimates of medication use between 2003 and 2030. The increase in medication costs was estimated to be $16.9 \%$, which was primarily accounted for by patients approaching death. In this regard, the findings of Kildemoes et al. [18] agree with those of this study.

Aplin et al. [19] studied the effects of patient-related variables on hospital costs. They collected data for 118 million hospital admissions from 2008 to 2010. Based on their results, length of stay, the number of procedures, major diagnostic categories, major operating room procedures, and ownership of the hospital were important variables affecting hospital costs. The results of our study are consistent with those findings. In our study, the type of disease (especially chronic conditions such as joint/orthopedic diseases), drugs, surgeons, and paraclinical services played important roles in hospital costs. Furthermore, the mean costs of the intensive care unit were higher than those of all other wards. Ziloochi et al. [3] similarly reported that the intensive care unit had the highest costs, and that bed-days were associated with the highest cost share. The costs of caring for the elderly is a fundamental aspect that should be taken into account in the health sector, as these costs include outpatient and inpatient treatment, medications, diagnostic tests, and maintenance care at home or a nursing home for the elderly. Since the elderly receive a significant share of healthcare services, the costs of services provided to this stratum is of vital importance from a health economics perspective. The growth in the elderly population will cause challenges for the sustainability of the welfare and health systems.

Societies can take action to overcome these challenges, and by doing so they have the opportunity to increase the likelihood of high-quality life years and high-health status and to improve the efficiency of systems based on an appropriate consideration of the needs of the elderly. Population-based interventions will be critical for promoting health and avoiding diseases, as well as confronting aging. The public promotion of positive lifestyle steps such as avoiding smoking and consumption of alcohol, promoting physical activity, consuming a proper diet, and controlling obesity as part of active aging will not only lead to an increase in life expectancy and the postponement of costs, but also consequent economic benefits. As some studies have shown, integrating palliative and hospice care into the health system may be a highly effective intervention for cost-saving in hospitals [20].

The limitations of the this study, we only studied elderly patients admitted to a single hospital in the city of Tehran, which may affect the generalizability of the results to the entire population. The studied hospital belongs to a military organization that mainly provides care for its members with low outof-pocket payments, which may pose problems for our initial assumption that there would be no induced demand or moral hazard.

The results showed that healthcare costs were higher for elderly patients approaching death, as the costs for patients who died were 3.9 times higher than those for persons discharged alive. For elderly people who suffer from chronic diseases, home care is recommended to reduce hospital costs.

\section{CONFLICT OF INTEREST}

The authors have no conflicts of interest associated with the material presented in this paper.

\section{FUNDING}

None.

\section{ACKNOWLEDGEMENTS}

None. 


\section{AUTHOR CONTRIBUTIONS}

Conceptualization: EH. Data curation: NMM, SZBJ. Funding acquisition: None. Methodology: ZM, SHB. Project administration: EH. Writing - original draft: EH, ZM, SHB. Writing - review \& editing: SZBJ, NMM.

\section{ORCID}

Ebrahim Hazrati https://orcid.org/0000-0002-6987-7404

Zahra Meshkani https://orcid.org/0000-0001-7994-2567

Saeed Husseini Barghzan https://orcid.org/0000-00025322-2438

Sanaz Zargar Balaye Jame https://orcid.org/0000-00018393-7314

Nader Markazi-Moghaddam https://orcid.org/0000-00032861-2765

\section{REFERENCES}

1. Lehnert T, Heider $D$, Leicht $H$, Heinrich $S$, Corrieri S, Luppa $M$, et al. Review: health care utilization and costs of elderly persons with multiple chronic conditions. Med Care Res Rev 2011; 68(4):387-420.

2. Ellen ME, Panisset U, de Carvalho IA, Goodwin J, Beard J. A knowledge translation framework on ageing and health. Health Policy 2017;121(3):282-291.

3. Ziloochi MH, Pourreza A, Akbari F, Rahimi-Foroshani A. Evaluating the hospitalization costs for elderly patients in teaching hospitals of Kashan university of medical sciences during 2009-10. J Kashan Univ Med Sci 2010;16(1):86-94 (Persian).

4. Jacobzone S. Coping with aging: international challenges. What are the implications of greater longevity and declining disability levels? Health Aff 2000;19(3):213-225.

5. Tsuji M, Miyahara S, Taoka F, Teshima M. An estimation of economic effects of tele-home-care: hospital cost-savings of the elderly. Stud Health Technol Inform 2001;84(Pt 1):858-862.

6. Prince MJ, Wu F, Guo Y, Gutierrez Robledo LM, O'Donnell M, Sullivan $\mathrm{R}$, et al. The burden of disease in older people and implications for health policy and practice. Lancet 2015;385(9967): 549-562.

7. Rolston KV. Polymicrobial pulmonary infections in cancer patients with underlying solid tumors. Infection 2017;45(2):245-
246.

8. Anderson GF, Hussey PS. Population aging: a comparison among industrialized countries. Health Aff (Millwood) 2000;19(3):191203.

9. Chan A, Chiang YY, Low XH, Yap KY, Ng R. Affordability of cancer treatment for aging cancer patients in Singapore: an analysis of health, lifestyle, and financial burden. Support Care Cancer 2013;21(12):3509-3517.

10. Tolsgaard MG, Tabor A, Madsen ME, Wulff CB, Dyre L, Ringsted $C$, et al. Linking quality of care and training costs: cost-effectiveness in health professions education. Med Educ 2015;49(12): 1263-1271.

11. Mendelson DN, Schwartz WB. The effects of aging and population growth on health care costs. Health Aff (Millwood) 1993; 12(1):119-125.

12. Reinhardt UE. Does the aging of the population really drive the demand for health care? Health Aff (Millwood) 2003;22(6): 27-39.

13. Brockmann $\mathrm{H}$. Why is less money spent on health care for the elderly than for the rest of the population? Health care rationing in German hospitals. Soc Sci Med 2002;55(4):593-608.

14. Hazra NC, Rudisill C, Gulliford MC. Determinants of health care costs in the senior elderly: age, comorbidity, impairment, or proximity to death? Eur J Health Econ 2018;19(6):831-842.

15. Frick KD. Micro-costing quantity data collection methods. Med Care 2009;47(7 Suppl 1):S76-S81.

16. Polder JJ, Barendregt JJ, van Oers H. Health care costs in the last year of life - the Dutch experience. Soc Sci Med 2006;63(7): 1720-1731.

17. Mcgrail K, Green B, Barer ML, Evans RG, Hertzman C, Normand C. Age, costs of acute and long-term care and proximity to death: evidence for 1987-88 and 1994-95 in British Columbia. Age Ageing 2000;29(3):249-253.

18. Kildemoes HW, Christiansen T, Gyrd-Hansen D, Kristiansen IS, Andersen M. The impact of population ageing on future Danish drug expenditure. Health Policy 2006;75(3):298-311.

19. Aplin B, Nazzal M, Qu W, Zelenock G, Kazan V, Abbas J. Patient variables impacting hospital costs from 2008 to 2010. Am J Surg 2015;210(1):134-140.

20. Morrison RS, Penrod JD, Cassel JB, Caust-Ellenbogen M, Litke A, Spragens $L$, et al. Cost savings associated with US hospital palliative care consultation programs. Arch Intern Med 2008; 168(16):1783-1790. 